\title{
Jóvenes universitarias y normalistas ante las jornadas de luchas feministas de marzo del 2020
}

Young University Students and Normalistas Before the Days of Feminist

Struggles in March 2020

Jovens universitárias e normalistas antes dos dias de lutas feministas em

março de 2020

Arturo Torres Mendoza

Universidad Pedagógica Nacional, Escuela Normal Superior de Jalisco, México

arturo.torres@upn141.edu.mx

https://orcid.org/0000-0003-0673-696X

\section{Resumen}

En este documento se resalta el posicionamiento de las estudiantes de diversas licenciaturas sobre cuestiones de género muy puntuales: el machismo, el acoso sexual, la agresión psicológica o física, el trato que reciben las mujeres en casa y la forma en que se reparten las tareas domésticas. El objetivo que se planteó fue conocer cómo se posicionan las estudiantes de las instituciones de educación superior respecto a las demandas feministas y las protestas del 8 y 9 de marzo del 2020. Las participantes fueron 290 estudiantes de las siguientes instituciones: Escuela Normal Superior de Jalisco (ENSJ), Universidad Pedagógica Nacional (UPN), Unidad 141 Guadalajara, y Universidad de Guadalajara (UdeG). Dentro de los resultados y conclusiones se destaca que una alta proporción de la muestra participó en las jornadas de lucha del Día Internacional de Mujer del 2020; la inmensa mayoría de la UdeG y de la ENSJ considera que existe discriminación y violencia en sus espacios de estudio, no así para las de la UPN; en todos los casos, los actos de violencia y discriminación disminuyeron con las clases virtuales, y respecto a la violencia 

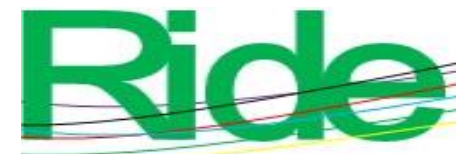
Investigación y el Desarrollo Educativo ISSN 2007 - 7467

intrafamiliar, la mayoría de las estudiantes de las tres instituciones niega que esté presente en casa, sin embargo, reconocen, en altos porcentajes, que las tareas domésticas las realizan, sobre todo, las mujeres.

Palabras clave: derechos de la mujer, discriminación, enseñanza superior, movimiento de liberación femenina, violencia doméstica.

\section{Abstract}

This document highlights the position of the students of various degrees on very specific gender issues: machismo, sexual harassment, psychological or physical aggression, the treatment women receive at home, and the way housework is divided. The objective that was set was to know how the students of higher education institutions position themselves with respect to feminist demands and the protests of March 8 and 9, 2020. The participants were 290 students from the following institutions: Escuela Normal Superior de Jalisco (ENSJ), Universidad Pedagógica Nacional (UPN), Unidad 141 Guadalajara, and Universidad de Guadalajara (UdeG). Among the results and conclusions, it is highlighted that a high proportion of the sample participated in the days of struggle of the International Women's Day of 2020; the vast majority of the UdeG and the ENSJ consider that there is discrimination and violence in their study spaces, not so for those of the UPN; in all cases, acts of violence and discrimination decreased with virtual classes, and regarding domestic violence, most of the students from the three institutions deny that it is present at home, however, they recognize, in high percentages, that housework is carried out, above all, by women.

Keywords: women's rights, discrimination, higher education, women's liberation movement, domestic violence. 


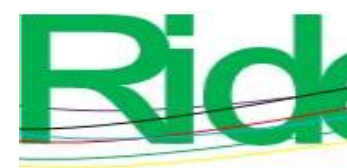

Revista Iberoamericana para la Investigación y el Desarrollo Educativo ISSN $2007-7467$

\section{Resumo}

Este documento destaca a posição dos estudantes de vários graus sobre questões de gênero muito específicas: machismo, assédio sexual, agressão psicológica ou física, o tratamento que as mulheres recebem em casa e a forma como as tarefas domésticas são distribuídas. O objetivo foi conhecer como as alunas das instituições de ensino superior se posicionam frente às demandas feministas e aos protestos de 8 e 9 de março de 2020. Participaram 290 alunos das seguintes instituições: Escuela Normal Superior de Jalisco (ENSJ ), Universidade Nacional Pedagógica (UPN), Unidade 141 Guadalajara e Universidade de Guadalajara (UdeG). Dentre os resultados e conclusões, destaca-se que grande parte da amostra participou dos dias de luta do Dia Internacional da Mulher de 2020; A grande maioria da UdeG e da ENSJ considera que existe discriminação e violência nos seus espaços de estudo, mas não para os da UPN; Em todos os casos, os atos de violência e discriminação diminuíram com as aulas virtuais e, no que diz respeito à violência doméstica, a maioria dos alunos das três instituições nega que esteja presente em casa, mas reconhecem, em percentagens elevadas, que o trabalho doméstico é realizado, acima de tudo, por mulheres.

Palavras-chave: direitos das mulheres, discriminação, ensino superior, movimento de libertação das mulheres, violência doméstica.

Fecha Recepción: Enero 2021

Fecha Aceptación: Julio 2021

\section{Introducción}

\section{Contextualización de las jornadas de lucha feminista del 8 y 9 de marzo de 2020}

A lo largo de la historia, las mujeres han sufrido de falta de equidad de condiciones y de derechos. Desafortunadamente, la presencia de prácticas discriminatorias y de actos de violencia por razones de género siempre han estado presentes. Y aun en los diferentes momentos históricos en los que se ha buscado la igualdad de derechos, el hombre ha sido el centro de atención. Por ejemplo, en la "Declaración de los Derechos del Hombre y del Ciudadano" de 1789 se consigna lo siguiente: "Los representantes del pueblo francés, constituidos en asamblea nacional (...) han resuelto exponer, en una declaración solemne, los derechos naturales, inalienables y sagrados del hombre” (Ramírez, 2015. p. 10). 


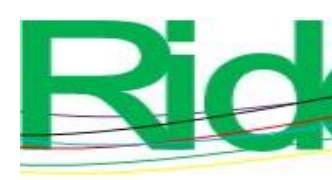

Revista Iberoamericana para la Investigación y el Desarrollo Educativo

ISSN 2007 - 7467

Frente a este invisibilidad, apenas un par de años después de la "Declaración de los Derechos del Hombre...", Olympe De Gouges redactó, a modo de respuesta, la "Declaración de los Derechos de la Mujer y de la Ciudadana", la cual fue presentada en la Asamblea Legislativa de Francia el 28 de octubre de 1791. Allí se establece, en su artículo 1, lo siguiente: "La mujer nace libre y permanece igual al hombre en derechos. Las distinciones sociales solo pueden estar fundadas en la utilidad común" (Ramírez, 2015, p. 7). Y antes, en el preámbulo, dice: "El hombre esclavo ha redoblado sus fuerzas y ha necesitado apelar a las tuyas para romper sus cadenas. Pero una vez en libertad, ha sido injusto con su compañera" (Ramírez, 2015, p. 7). No obstante, a pesar del esfuerzo de Olympe De Gouges de incorporar el reconocimiento de las mujeres, lo que prevaleció fue el contenido del documento de la asamblea francesa de 1789.

En el siglo XIX, las mujeres protagonizaron numerosas luchas por la igualdad de género. Destaca la Declaración de Seneca Falls (1848), ${ }^{1}$ documento resultado de una reunión de diversos movimientos y asociaciones, lideradas por Lucretia Mott y Elizabeth Cady Stanton, para discutir las condiciones, derechos sociales, civiles y religiosos de las mujeres. "La historia de la humanidad es la historia de las repetidas vejaciones y usurpaciones perpetradas por el hombre contra la mujer, con el objetivo directo de establecer una tiranía absoluta sobre ella" (párr. 4). Entre otras cosas, el documento reivindica el derecho de las mujeres al sufragio, el acceso a la educación universitaria, la igualdad en el trabajo y la remuneración justa. Al mismo tiempo que se pugnaba por la igualdad en el terreno de las ideas, las obreras europeas y americanas libraron varias batallas en pro de sus derechos laborales y del sufragio.

Como se advierte, desde el siglo XIX las mujeres lucharon por la conquista de derechos sindicales, de reunión y de acceso al sufragio. En los inicios del siglo XX, los dos primeros ya habían sido conquistados, sin embargo, en muchos países del orbe, el derecho a votar y ser votadas aún estaba pendiente. Fue después de la Segunda Guerra Mundial, el 10 de diciembre de 1948, cuando la Organización de las Naciones Unidas (ONU) y los Estados miembros de la Asamblea General de la ONU ratificaron la "Declaración Universal de Derechos Humanos". Allí, en el preámbulo, se menciona lo siguiente: "La libertad, la justicia y la paz en el mundo tienen por base el reconocimiento de la dignidad intrínseca y de los derechos iguales e inalienables de todos los miembros de la familia humana" (párr.

\footnotetext{
${ }^{1}$ A esta declaración se le considera el texto fundacional del movimiento social feminista.
} 


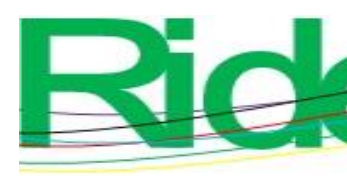

Revista Iberoamericana para la Investigación y el Desarrollo Educativo ISSN 2007 - 7467

1). También se considera "esencial que los derechos humanos sean protegidos por un régimen de Derecho, a fin de que el hombre no se vea compelido al supremo recurso de la rebelión contra la tiranía y la opresión" (párr. 3). Y finalmente, se señala que "los pueblos de las Naciones Unidas han reafirmado en la Carta su fe (...) en la igualdad de derechos de hombres y mujeres" (párr. 5). Si bien es cierto que en el párrafo tercero se refiere al hombre, en el previo se habla de la familia humana y en el quinto se menciona la necesidad de lograr la igualdad entre hombres y mujeres, por lo que ya existe un expreso reconocimiento a la igualdad de género.

Más adelante, la propia ONU (1976) declaró a 1975 como el Año Internacional de la Mujer y enfatizó en que la discriminación contra la mujer es incompatible con la dignidad humana. Aun con todo, se debe "intensificar la acción con miras a: promover la igualdad entre hombres y mujeres" (p. 2). Todo lo hasta aquí mencionado deja en claro que gracias a las luchas feministas hoy en día las mujeres gozan de derechos como el de asociación, manifestación, sindicalización y sufragio.

Al día de hoy, si bien se goza de las victorias arriba mencionadas, la brecha de desigualdad de género no se ha acortado del todo, y las mujeres continúan organizándose con el objetivo de ser finalmente escuchadas. Y una de las formas que han encontrado para alzar la voz han sido las movilizaciones realizadas año con año el 8 de marzo, fechada declarada Día Internacional de la Mujer. El año pasado, poco antes de que la propagación del coronavirus del 2019 se declarara oficialmente como pandemia, se vivió uno de los 8 de marzo con mayor efervescencia en México y gran parte de los países latinoamericanos.

Enseguida se habla, en principio, de las movilizaciones del 8 de marzo de 2020, con énfasis en las que se sucedieron en América Latina, para luego acercar más el lente y hacer referencia a las que se dieron el día 8 y 9 en nuestro país, primeramente, y más tarde en Jalisco, y finalmente concluir con la percepción de algunos estudiantes de instituciones de educación superior de Guadalajara sobre el tópico y la jornada en cuestión. Los datos que a continuación se consignan se han tomado de un reportaje publicado en el periódico digital español El País firmado por varias autoras (Corona et al., 8 de marzo de 2020).

El reportaje, titulado "Miles de mujeres exhiben su fuerza en las calles de América Latina (Corona et al., 8 de marzo de 2020), explica que el creciente descontento de las mujeres de América Latina llevó a millones de ellas a manifestarse en la mayoría de países de la región y a pintar las calles de verde y morado, colores representativos de la lucha 


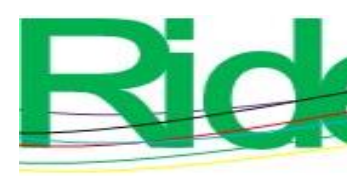

Revista Iberoamericana para la Investigación y el Desarrollo Educativo

ISSN 2007 - 7467

feminista. Entre los países que resaltaron por el número de participantes se encuentran Argentina, Chile y México.

En el caso de Chile, fueron más de dos millones de mujeres las que salieron a protestar a las calles; de ellas, 125000 se manifestaron en la ciudad de Santiago. Acudieron de todas las generaciones pero sobre todo jóvenes. En las movilizaciones estuvieron presentes algunos símbolos de lucha, entre ellos el pañuelo verde, aporte de las argentinas, y las capuchas rojas, contribución de las chilenas. También se llevaron a cabo instalaciones artísticas como El violador eres tú del colectivo feminista Lastesis. Por supuesto, se recordó a las mujeres víctimas de feminicidio con un minuto de silencio (Corona et al., 8 de marzo de 2020). En cuanto a las demandas puntuales, destacaba la que exige la aprobación de la ley que termina con la discriminación entre hombres y mujeres en la administración de la sociedad conyugal, además de pedir combatir el asesinato de mujeres por odio.

En Argentina, si bien las protestas se trasladaron al 9 de marzo, un día antes se convocó “a un pañuelazo frente a la catedral de Buenos Aires a favor del aborto legal, seguro y gratuito para contestar a la misa convocada en contra por la Iglesia católica argentina en el principal santuario del país" (Corona et al., párr. 10). Esto a raíz de que el presidente Alberto Fernández estaba por enviar un proyecto de ley al respecto al congreso para su discusión y aprobación. Así pues, la principal demanda fue la aprobación de una ley de plazos para la interrupción legal del embarazo, que ya se ha conquistado en Uruguay, Cuba, Guyana y la capital mexicana.

En México, se prepararon dos grandes eventos de protesta: las manifestaciones para el 8 de marzo y un paro convocado para el siguiente día, es decir, el lunes 9. Este último fue denominado en la redes sociales como $9 M$ y difundido a través del hashtag \#ElNueveNadieSeMueve. Se trataba de que todas las mujeres, trabajadoras de la industria, del comercio, de los servicios, burócratas, profesoras, estudiantes y amas de casa, no se presentaran a trabajar o estudiar y no hicieran nada ese día con el propósito de demostrar la importancia de las mujeres, exigir un trato igualitario y sobre todo protestar contra los feminicidios (Topete, 20 de febrero de 2020).

En cuanto al paro del lunes 9 de marzo, Topete (20 de febrero de 2020), que entrevistó a Lupita Ramos, coordinadora del Comité de América Latina y El Caribe para la Defensa de los Derechos de la Mujer en México (Cladem), manifestó que la iniciativa 


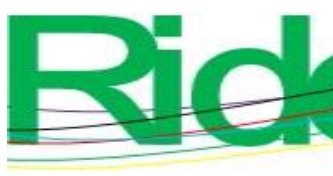

Revista Iberoamericana para la Investigación y el Desarrollo Educativo

ISSN 2007 - 7467

“surgió en el Encuentro Feminista Latinoamericano de 2016 que tuvo sede en Montevideo, Uruguay. La iniciativa propone hacer huelga general de mujeres” (párr. 2).

En Jalisco, se unieron diferentes colectivos feministas y de familiares de desaparecidos para la protesta del día 8 de marzo: "Me cuidan mis amigas Gdl, Femibici, Vulvurina, Lavanda, Cetien México, Antimusas, Batukada Feminista, Cladem Jalisco, Género y Lucha de Clases, y Cuerpos parlantes espacio feminista y de investigación urbana" (Topete, 20 de febrero de 2020, párr. 3).

\section{El problema de investigación}

Siguiendo lo consignado líneas arriba, hemos visto que las mujeres han sido objeto de discriminación y violencias de parte de los hombres. Sin duda se trata de un problema de carácter estructural. En las últimas cinco décadas, sin haber desaparecido algunas demandas añejas, han surgido otras, entre ellas la eliminación del machismo, la igualdad plena de género, la lucha contra el feminicidio y las violencias sexuales, el reconocimiento del trabajo en casa y el derecho al aborto universal, seguro y gratuito.

El estudio que aquí se presenta es diferente a muchos de los hasta ahora publicados dentro de la línea de investigación en que se inscribe en cuanto a los propósitos y las participantes, lo cual se verá claramente más adelante. Por si fuera poco, este esfuerzo investigativo coincidió con el confinamiento por la pandemia de COVID-19, lo que, no está de más señalar, alejó a las chicas de su rutina diaria pero no de las conductas de discriminación y violencia a las que son sujetas.

A raíz de la relación educativa que se tiene con las estudiantes de algunas instituciones de educación superior del estado de Jalisco, hemos escuchado algunas de las prácticas discriminatorias que padecen dentro del ámbito académico. Sobre todo destaca que refieren trato machista por parte de profesores y estudiantes. Entonces, para conocer aún más el grado de información y el involucramiento que tienen respecto a las luchas y movilizaciones por los derechos de las mujeres y el nivel en que perciben la discriminación y las violencias ejercidas hacia ellas, y teniendo como referente las movilizaciones del 8 y 9 de marzo, se construyó la siguiente pregunta de investigación: ¿cómo se posicionan las estudiantes de las instituciones de educación superior de Guadalajara ante las reivindicaciones de las mujeres por sus derechos en el marco de las protestas de marzo del 2020? En cuanto a la hipótesis general, se formuló de esta manera: las estudiantes de las 


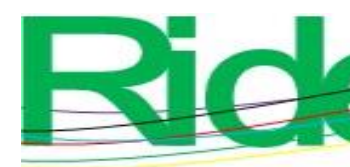

Revista Iberoamericana para la Investigación y el Desarrollo Educativo ISSN 2007 - 7467

instituciones de educación superior están informadas sobre los derechos adquiridos como mujeres y los reivindican en los espacios escolares y familiares.

\section{Apartado teórico-conceptual y metodológico Apartado teórico-conceptual}

Para el abordaje del apartado teórico-conceptual, se partió de las ideas de Bourdieu (2000), quien considera que la visión androcéntrica da lugar a la dominación masculina, la cual se impone, sobre todo, mediante la violencia simbólica, que a su vez implica la adhesión del dominado a los requerimientos del dominador, por tanto, se acepta de manera tácita la dominación que conlleva a la sumisión.

Además, la violencia simbólica no está solo en la piel, no únicamente en sus conciencias, se encuentra en estructuras de dominación que la prohíjan, por lo que el cambio es posible si se modifican las condiciones sociales que permiten su reproducción, sobre todo si se considera que es en la familia donde se presenta e incuba la dominación y visión masculina (Bourdieu, 2000).

Por otro lado, si la dominación masculina ha sido puesta en cuestión, ello se debe al trabajo de los movimientos feministas de carácter crítico, que han ido rompiendo los espacios de dominación, los cuales han sido erosionados por cambios en la nueva condición femenina, sobre todo en las capas sociales más favorecidas social y económicamente, que han tenido acceso al estudio, al trabajo remunerado, a su acceso a la esfera pública, al tiempo que se apartan de las labores domésticas, disminuyen la nupcialidad y retrasan la procreación (Bourdieu, 2000).

Lamas (1994) comparte la idea de que la dominación masculina se lleva a cabo por vía de la violencia simbólica. Con el objetivo de clarificar su propuesta teórica, centra su atención en el concepto de género. Este es considerado una construcción social que se basa en las características femeninas o masculinas que se les atribuyen a los sexos; a partir de ello, se han construido estereotipos de lo que corresponde hacer y manera de comportarse hombres o mujeres. Se trata, por tanto, de ideas socialmente fabricadas. Por lo que el discurso feminista tiene como tarea criticar y llevar a efecto la deconstrucción de prácticas y representaciones sociales de carácter opresor para lograr una redefinición de lo que es ser persona, sujeto, ser humano. 


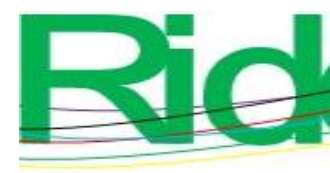

Revista Iberoamericana para la Investigación y el Desarrollo Educativo

ISSN 2007-7467

Buquet, Cooper, Mingo y Moreno (2013), por su parte, plantean que el problema fundamental de la desigualdad entre los sexos radica en la violencia simbólica. A partir de estudios que han realizado, se dieron cuenta de que las mujeres, ante hechos de hostigamiento sexual, no toman acciones de defensa o denuncia, ya que no le dan importancia, con lo que se acepta la normalización a la dominación masculina y las desigualdades por ser "naturales" y formar parte de la vida cotidiana, de ahí que se legitime la violencia cometida hacia las mujeres.

Asimismo, Buquet et al. (2013) refieren que las universitarias mujeres dedican más tiempo que sus colegas varones a las tareas del hogar, ya que los hombres participan menos en las tareas domésticas y familiares, por lo que se requiere de un gran esfuerzo de transformación cultural que elimine la violencia simbólica para que las tareas de casa y la atención a la familia sean de corresponsabilidad y no exclusivamente de las dominadas.

Mientras que Castro (2020) considera que existe un discurso sexista entre estudiantes universitarios; sostiene que lo imperante es la denominada masculinidad hegemónica, entendida como una estrategia que legitima el patriarcado y da poder al hombre sobre la mujer, y que eso tiene su sustento en procesos psicosociales, aunque la violencia y el sexismo no tienen solo ese fundamento.

Por último, vale la pena mencionar el trabajo de Esquivel (2019), quien, a partir de una revisión de las redes sociales, particularmente en hashtags, analiza las relaciones de poder tanto en el ámbito privado como público que reproducen las desigualdades de género en la política, la ciencia y la academia, y advierte que una de las características de las protestas feministas en las redes es lograr la visibilización de las experiencias de violencia que sufren las mujeres.

De lo escrito en los párrafos anteriores, se puede decir que los conceptos e ideas que guían teóricamente esta entrega son: la familia como espacio de reproducción de estereotipos de género, dominación masculina, papel del feminismo, desempeño de tareas domésticas y familiares y, desde luego, la violencia simbólica. 


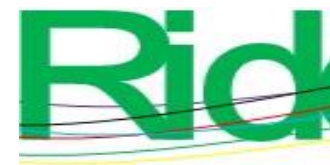

Revista Iberoamericana para la Investigación y el Desarrollo Educativo ISSN $2007-7467$

\section{Método}

La presente investigación se abordó con un enfoque descriptivo, no experimental, mediante un estudio de encuesta (Torrado, 2009). Se elaboró un cuestionario para la recogida de los datos denominado La opinión de las universitarias y normalistas ante las jornadas de lucha de las mujeres de marzo de 2020, el cual fue aplicado a 290 estudiantes del sexo femenino.

\section{Diseño del instrumento}

La técnica que se utilizó, en consonancia con Torrado (2009), fue la encuesta. Para ello, se elaboró un instrumento compuesto por 16 ítems de opción múltiple distribuidos en tres apartados. En la primera sección (1-5) se utilizaron preguntas de opción múltiple para indagar sobre los datos generales de las estudiantes y la institución de adscripción; en la segunda (6-12), se indaga sobre su información, forma de participación en las jornadas de lucha de marzo del 2020 y su opinión sobre la violencia de género en su institución educativa; y en la tercera (13-16), sobre el trato que reciben en su casa y quiénes realizan las tareas domésticas en tiempos de confinamiento.

\section{Validación del instrumento}

Se evaluó la claridad y pertinencia de las preguntas con respecto al objeto de estudio. En esta tarea participaron dos profesoras de la especialidad en Estudios de Género de la Universidad Pedagógica Nacional, además de realizar un grupo de discusión con estudiantes mujeres de la UPN, lo que permitió mejorar la redacción de los ítems. También se solicitó la inclusión del correo electrónico de todas las participantes para evitar que se respondiera el cuestionario más de una ocasión por una misma persona.

\section{Universo y selección de la muestra}

El universo de aplicación del instrumento lo comprendieron las estudiantes de las siguientes instituciones de educación superior: Universidad de Guadalajara (UdeG), la UPN Unidad 141 Guadalajara y la Escuela Normal Superior de Jalisco (ENSJ). La muestra fue intencional. La selección se dio por muestreo no probabilístico, considerando la 

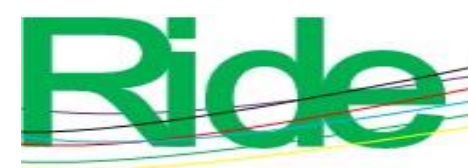

Revista Iberoamericana para la Investigación y el Desarrollo Educativo

ISSN 2007 - 7467

accesibilidad, donde el requisito era ser mujer y estudiante. En total, hubo una participación de 290 sujetos de estudio.

Las edades de las estudiantes estuvieron comprendidas entre los 18 y 40 años, no obstante, la inmensa mayoría quedó comprendida entre los 18 y 25 años (90 \% de la muestra). La fecha de aplicación se dio entre el 15 y el 25 de marzo de 2020, mediante Google Form, por lo que fue realizado en línea.

\section{Sobre el apoyo en la aplicación de la encuesta}

Para la aplicación de las encuestas se contó con el apoyo de las representantes estudiantiles de tres grupos de la licenciatura en Pedagogía de la UPN Unidad 141 Guadalajara, de la coordinadora de investigación de la ENSJ y de dos alumnas de la UdeG, que solicitaron por vía de sus redes sociales universitarias la participación de estudiantes mujeres de dicha institución educativa.

\section{Procesamiento de la información}

Para procesar los datos recogidos, se descargó la hoja de matriz de información del formulario electrónico y se migró al software Microsoft Excel, que permitió su tratamiento estadístico y la elaboración de tablas que contienen los datos que aquí se exponen.

\section{Resultados}

La primera pregunta que se formuló fue sobre su edad. La inmensa mayoría son jóvenes universitarias de entre 18 y 25 años. De las 290 participantes, 262 se encuentran en este rango de edad, lo que representa $90 \%$ del total de la muestra. En cuanto al resto, 22 tienen entre 26 y 30 años (8\%) y solo seis tienen entre 31 y 40 años (2\%).

La segunda pregunta versó sobre la institución de educación superior en la que se encuentran estudiando. El mayor número de participantes provenían de la UdeG, enseguida las de la UPN y al final las de la ENSJ. Se entiende, ya que la primera es una institución que alberga miles de estudiantes de licenciatura, la segunda tiene una población mayoritariamente femenina y la tercera es una institución con menos estudiantes mujeres que las anteriores. 


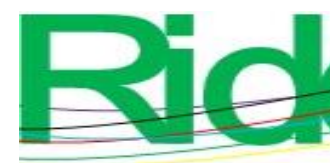

\section{Revista Iberoamericana para la Investigación y el Desarrollo Educativo ISSN 2007 - 7467}

Cuando se les preguntó sobre el medio mediante el cual se enteraron de las manifestaciones feministas del 8 y 9 de marzo de 2020, 269, es decir, $93 \%$, se informa por las redes sociales, lo que habla de la fuerte conexión que tienen las chicas con la tecnología y que esta les permite enterarse en tiempo real de los acontecimientos que les interesan.

Cuando se les cuestionó sobre la forma en que participaron en las jornadas de lucha feminista encontramos que como organizadora solo lo hizo una chica, sin embargo, fueron 238 quienes tuvieron una participación activa, sea en foros, marchas, difusión en redes o platicando con familiares, amigos y conocidos; en conjunto, representan $82 \%$, y solo 51 , esto es, $18 \%$, no participaron de ninguna manera, lo cual indica que más de 8 de cada 10 se vieron involucradas de alguna manera. Los números por institución educativa se incluyen en la siguiente tabla.

Tabla 1. Forma de participación

\begin{tabular}{|l|c|c|c|c|c|c|}
\hline \multicolumn{1}{|c|}{$\begin{array}{c}\text { Forma de } \\
\text { participación }\end{array}$} & \multicolumn{2}{c|}{ UPN } & \multicolumn{2}{c|}{ ENSJ } & \multicolumn{2}{c|}{} \\
\hline & Cantidad & porcentaje & Cantidad & Porcentaje & Cantidad & Porcentaje \\
\hline Organizadora & 1 & $1.1 \%$ & - & $0 \%$ & - & $0 \%$ \\
\hline Foros y marchas & 6 & $7.1 \%$ & 3 & $6 \%$ & 51 & $32.6 \%$ \\
\hline $\begin{array}{l}\text { Difusión en las } \\
\text { redes }\end{array}$ & 25 & $29.6 \%$ & 13 & $26 \%$ & 47 & $30.1 \%$ \\
\hline $\begin{array}{l}\text { Platicando con } \\
\text { familiares, amigos } \\
\text { y conocidos }\end{array}$ & 34 & $40.4 \%$ & 25 & $50 \%$ & 48 & $30.7 \%$ \\
\hline De ninguna forma & 24 & $28.5 \%$ & 9 & $18 \%$ & 10 & $6.4 \%$ \\
\hline
\end{tabular}

Fuente: Elaboración propia

Las estudiantes de la UdeG, de acuerdo con las respuestas, tuvieron una gran participación en los foros y marchas, con más de $32 \%$, contra $7 \%$ y $6 \%$ de las chicas de la UPN y la ENSJ.

A continuación se muestran los resultados al cuestionamiento ¿En la institución donde estudias se presenta algún tipo de discriminación y violencia contra las estudiantes? La mayoría, esto es, $64 \%$, considera que existe discriminación y violencia contra las 


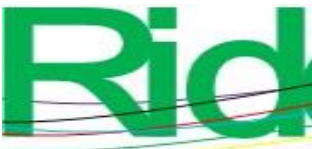

Revista Iberoamericana para la Investigación y el Desarrollo Educativo

ISSN 2007 - 7467

jóvenes universitarias, contra $36 \%$ que opina lo contrario. En la tabla 2 se muestran los resultados por institución educativa.

Tabla 2. Presencia o no de discriminación y violencia

\begin{tabular}{|c|c|c|c|c|c|c|}
\hline $\begin{array}{c}\text { Se presenta la } \\
\text { discriminación y } \\
\text { violencia }\end{array}$ & \multicolumn{2}{|c|}{ UPN } & \multicolumn{2}{c|}{ ENSJ } & \multicolumn{2}{c|}{} \\
\hline & Cantidad & Porcentaje & Cantidad & Porcentaje & Cantidad & Porcentaje \\
\hline Sí & 17 & $20.2 \%$ & 35 & $70 \%$ & 124 & $79.4 \%$ \\
\hline No & 67 & $79.7 \%$ & 15 & $30 \%$ & 22 & $14.1 \%$ \\
\hline
\end{tabular}

Fuente: Elaboración propia

Se advierte una gran desproporción entre los porcentajes, pues mientras las estudiantes de la UdeG y de la ENSJ señalan que se presenta la discriminación y la violencia en sus escuelas, con $79 \%$ y $70 \%$ respectivamente, solo una de cada cinco alumnas de la UPN así lo considera.

Frente a la pregunta ¿Qué tipo de discriminación o violencia hacia las mujeres se presentan en tu institución educativa de parte de tus compañeros estudiantes? Para 111, no existió ningún tipo de discriminación o violencia de parte de sus compañeros estudiantes, pero el resto menciona utilización de lenguaje machista, con 130 menciones, acoso sexual, con 102, agresión psicológica, con 52; para el caso de todas, hubo 28 registros e incluso siete alumnas respondieron agresión física. Por institución educativa se visualizan datos en la tabla 3 . 
Tabla 3. Formas de discriminación o violencia por institución

\begin{tabular}{|c|c|c|c|}
\hline $\begin{array}{l}\text { Tipo de discriminación y/o } \\
\text { violencia }\end{array}$ & UPN & ENSJ & UdeG \\
\hline & $\begin{array}{l}\text { Cantidad de } \\
\text { menciones }\end{array}$ & $\begin{array}{l}\text { Cantidad de } \\
\text { menciones }\end{array}$ & $\begin{array}{l}\text { Cantidad de } \\
\text { menciones }\end{array}$ \\
\hline Utilización de lenguaje machista & 3 & 20 & 103 \\
\hline Acoso sexual & 1 & 15 & 86 \\
\hline Agresión física & - & 2 & 5 \\
\hline Agresión psicológica & 5 & 9 & 38 \\
\hline Todas las anteriores & - & 2 & 26 \\
\hline Ninguna de las mencionadas & 71 & 4 & 36 \\
\hline
\end{tabular}

Fuente: Elaboración propia

Se ve la diferencia entre los resultados de las estudiantes de la UPN y las otras dos instituciones, ya que la inmensa mayoría de chicas upenianas consideró que no son discriminadas o violentadas por sus compañeros. Quizá las respuestas se deban a que en la licenciatura en Pedagogía, a la que pertenecen las encuestadas de la UPN, más de $90 \%$ de la matrícula está compuesta por mujeres.

Para la pregunta ¿Qué tipo de discriminación o violencia hacia las mujeres se presentan en tu institución educativa de parte tus profesores o autoridades escolares en general?, 104 consideran que no existe discriminación o violencia de ningún tipo, pero disminuye sensiblemente en cuanto a la opción que involucra todas las respuestas, y lo mismo pasa en relación con la agresión física, donde solo existieron dos menciones. Enseguida los resultados por institución educativa. 


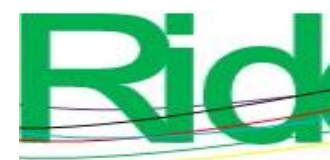

Revista Iberoamericana para la Investigación y el Desarrollo Educativo ISSN 2007 - 7467

Tabla 4. Discriminación o violencia de parte de profesores o autoridades escolares

\begin{tabular}{|l|c|c|c|}
\hline $\begin{array}{l}\text { Tipo de discriminación o } \\
\text { violencia }\end{array}$ & UPN & ENSJ \\
\hline & $\begin{array}{c}\text { Cantidad de } \\
\text { menciones }\end{array}$ & $\begin{array}{c}\text { Cantidad de } \\
\text { menciones }\end{array}$ & $\begin{array}{c}\text { Cantidad de } \\
\text { menciones }\end{array}$ \\
\hline Utilización de lenguaje machista & 2 & 16 & 116 \\
\hline Acoso sexual & 1 & 26 & - \\
\hline Agresión física & - & - & 107 \\
\hline Agresión psicológica & 7 & 12 & 25 \\
\hline Todas las anteriores & - & 9 & 2 \\
\hline Ninguna de las mencionadas & 70 & 2 & 25 \\
\hline
\end{tabular}

Fuente: Elaboración propia

Como en el caso anterior, pero ahora refiriéndose a sus profesores y las autoridades escolares, la inmensa mayoría de las estudiantes de la UPN, a diferencia de las de la ENSJ y de la UdeG, no se sienten discriminadas o violentadas, sin embargo, ahora no se puede suponer que se deba a que la mayor parte de la matrícula sea de mujeres.

Aquí se planteó, como consecuencia del confinamiento no estás asistiendo a clases presenciales pero siguen su curso las actividades escolares de manera virtual. En la nueva situación, ¿Se presentan las siguientes actitudes por parte de tus compañeros estudiantes?: utilización de lenguaje machista, acoso sexual, agresión física, agresión psicológica, todas las anteriores o ninguna de las mencionadas. Resultó que en tiempos de confinamiento y clases en línea las chicas se sienten menos agredidas por sus compañeros estudiantes, ya que 243 menciones apuntan que no se presenta discriminación o violencia hacia ellas; no obstante, sigue presente la utilización del lenguaje machista, con 42 registros, agresión psicológica, con nueve, y una mención incluye todas las anteriores; ninguna se registró para la agresión física. Los datos por institución educativa se incluyen enseguida. 
Tabla 5. Discriminación o violencia de parte de estudiantes en tiempos de confinamiento

\begin{tabular}{|l|c|c|c|}
\hline $\begin{array}{l}\text { Tipo de discriminación y/o violencia } \\
\text { en tiempos de confinamiento }\end{array}$ & UPN & ENSJ \\
\hline $\begin{array}{l}\text { Tipo de discriminación y/o violencia } \\
\text { en tiempos de confinamiento }\end{array}$ & $\begin{array}{c}\text { Cantidad de } \\
\text { menciones }\end{array}$ & $\begin{array}{c}\text { Cantidad de } \\
\text { menciones }\end{array}$ & $\begin{array}{c}\text { Cantidad de } \\
\text { menciones }\end{array}$ \\
\hline Utilización de lenguaje machista & - & 3 & 39 \\
\hline Acoso sexual & - & 1 & 6 \\
\hline Agresión física & - & - & - \\
\hline Agresión psicológica & 1 & - & 1 \\
\hline Todas las anteriores & 83 & 46 & 114 \\
\hline Ninguna de las mencionadas & - & - & 7 \\
\hline
\end{tabular}

Fuente: Elaboración propia

Como se puede advertir, de nueva cuenta, las chicas de la UPN, a diferencia de sus pares de las otras dos instituciones, se consideran prácticamente libres de discriminación o violencia en tiempos de confinamiento, pues solo 1 de 84 señala que eso sí sucede.

Luego se formuló esta pregunta, como consecuencia del confinamiento no estás asistiendo a clases presenciales pero siguen su curso las actividades escolares de manera virtual, en la nueva situación, ¿Se presentan las siguientes actitudes por parte de tus profesores?: Utilización de lenguaje machista, acoso sexual, agresión física, agresión psicológica, todas las anteriores o ninguna de las mencionadas.

Aquí la inmensa mayoría dice que no recibieron ningún tipo de discriminación o violencia en las relaciones virtuales, no obstante, la utilización del lenguaje machista tiene nueve menciones, nueve para agresión psicológica y dos de acoso sexual. La tabla 6 contiene los datos por institución. 
Tabla 6. Discriminación o violencia de profesores en tiempos de confinamiento por institución educativa

\begin{tabular}{|l|c|c|c|}
\hline & \multicolumn{1}{|c|}{ UPN } & ENSJ & UdeG \\
\hline $\begin{array}{l}\text { Tipo de discriminación o violencia en } \\
\text { tiempos de confinamiento }\end{array}$ & $\begin{array}{l}\text { Cantidad de } \\
\text { menciones }\end{array}$ & $\begin{array}{l}\text { Cantidad de } \\
\text { menciones }\end{array}$ & $\begin{array}{l}\text { Cantidad de } \\
\text { menciones }\end{array}$ \\
\hline Utilización de lenguaje machista & - & - & 30 \\
\hline Acoso sexual & - & - & 2 \\
\hline Agresión física & 1 & 2 & 6 \\
\hline Agresión psicológica & - & - & 2 \\
\hline Todas las anteriores & 83 & 47 & 116 \\
\hline Ninguna de las mencionadas & - & 1 & 6 \\
\hline
\end{tabular}

Fuente: Elaboración propia

En esta ocasión, tanto las estudiantes de la ENSJ como las de la UPN, en su inmensa mayoría, se sientes libres de discriminación y violencia, en tanto que las de la UdeG, en una proporción nada despreciable, señalan lo contrario.

En relación con el ítem "Como consecuencia del confinamiento en tu hogar se han presentado casos de violencia familiar ejercida por...": los dos padres, papá, mamá, hermanos o ninguno de los anteriores. Para 241, esto es, $83 \%$, no existe la violencia intrafamiliar. Para la respuesta que involucra a ambos padres, tenemos 14 menciones, $5 \%$; 16 respondieron que por parte del papá (6\%), y solo dos registros señalan a la mamá (1\%). Aquí algo que llama la atención es que las respuestas que involucran a hermanos ocupan el primer lugar, con 17 casos (6 \%). La tabla 7 contiene los datos por institución educativa. 
Tabla 7. Confinamiento y violencia intrafamiliar

\begin{tabular}{|l|c|c|c|c|c|c|}
\hline $\begin{array}{l}\text { Violencia } \\
\text { ejercida por: }\end{array}$ & \multicolumn{3}{|c|}{ UPN } & \multicolumn{2}{c|}{ ENSJ } & \multicolumn{2}{c|}{ UdeG } \\
\hline & Cantidad & porcentaje & Cantidad & Porcentaje & Cantidad & Porcentaje \\
\hline Los dos padres & 1 & $1.19 \%$ & 1 & $2 \%$ & 12 & $7.69 \%$ \\
\hline Papá & 1 & $1.19 \%$ & 4 & $8 \%$ & 11 & $7 \%$ \\
\hline Mamá & - & $0 \%$ & - & $0 \%$ & 2 & $1.28 \%$ \\
\hline Hermanos & 5 & $5.9 \%$ & 1 & $2 \%$ & 11 & $7 \%$ \\
\hline $\begin{array}{l}\text { Ninguno de los } \\
\text { anteriores }\end{array}$ & 77 & $91.6 \%$ & 44 & $88 \%$ & 120 & $76.9 \%$ \\
\hline
\end{tabular}

Fuente: Elaboración propia

En el caso de institución educativa se puede apreciar que las estudiantes de la ENSJ y la UPN son las menos violentadas, en tanto que las de la UdeG señalan que lo son en un porcentaje considerable.

Para la pregunta “¿Qué trato dan tus padres a hijos e hijas?”: trato igualitario, dan preferencia a los varones, dan preferencia a las mujeres o no aplica por ser solo hijas mujeres. En la inmensa mayoría de casos se refiere un trato igualitario: 203 registros (70 $\%)$. Para seis (2\%), no aplica por vivir solo mujeres en el hogar. En tres casos se ven favorecidas las mujeres (solo $1 \%$ de la muestra). Y es revelador que, en el caso de los varones, reciben un mejor trato según 78 registros, esto es, $27 \%$. En la tabla 8 se consignan los datos por institución educativa. 


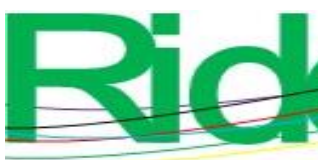

Revista Iberoamericana para la Investigación y el Desarrollo Educativo

ISSN 2007 - 7467

Tabla 8. El trato de padres a hijos

\begin{tabular}{|c|c|c|c|c|c|c|}
\hline Trato de los & \multicolumn{2}{|c|}{ UPN } & \multicolumn{2}{|c|}{ ENSJ } & \multicolumn{2}{|c|}{ UdeG } \\
\hline & Cantidad & Porcentaje & Cantidad & Porcentaje & Cantidad & Porcentaje \\
\hline Trato igualitario & 55 & $65.4 \%$ & 34 & $68 \%$ & 126 & $80.7 \%$ \\
\hline $\begin{array}{l}\text { Dan preferencia } \\
\text { a los varones }\end{array}$ & 11 & $13 \%$ & 7 & $14 \%$ & 16 & $10.2 \%$ \\
\hline $\begin{array}{l}\text { Dan preferencia } \\
\text { a las mujeres }\end{array}$ & 1 & $1.2 \%$ & - & $0 \%$ & - & $0 \%$ \\
\hline $\begin{array}{l}\text { No aplica por } \\
\text { ser solo hijas } \\
\text { mujeres }\end{array}$ & 17 & $20 \%$ & 9 & $18 \%$ & 14 & $8.9 \%$ \\
\hline
\end{tabular}

Fuente: Elaboración propia

En este caso, las estudiantes de la UPN y ENSJ dan valores similares. Sin embargo, no hay que dejar de señalar que, en una proporción considerable, los varones reciben mejor trato y llama la atención que las chicas de la UdeG hablan de trato igualitario en más de 80 $\%$ de los casos.

Para esta pregunta, con confinamiento o sin él en casa existen tareas domésticas, ¿Quién las realiza en este tiempo de confinamiento?: hombres y mujeres de manera igualitaria, hombres y mujeres pero más las mujeres, hombres y mujeres pero más los hombres, solo las mujeres de la casa, solo los hombres de la casa o no aplica, solo vivimos mujeres en casa

Solo 75 respuestas, que representan $26 \%$ del total, señalan que las tareas domésticas en tiempos de confinamiento las realizan de manera igualitaria hombres y mujeres. Nadie mencionó que solo los hombres. Hombres y mujeres pero más las mujeres registró 87 puntos (30 \%) y sólo las mujeres de la casa registró 55, esto es, $19 \%$. Lo anterior indica que las mujeres, en cualquier condición, son las que cargan principalmente con las tareas hogareñas. Enseguida, se incluyen los datos por institución. 


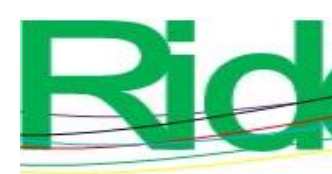

Revista Iberoamericana para la Investigación y el Desarrollo Educativo ISSN 2007 - 7467

Tabla 9. Las tareas domésticas en tiempos de confinamiento

\begin{tabular}{|l|c|c|c|c|c|c|}
\hline & \multicolumn{2}{|c|}{ UPN } & \multicolumn{2}{c|}{ ENSJ } & \multicolumn{2}{c|}{ UdeG } \\
\hline $\begin{array}{l}\text { Las tareas domésticas } \\
\text { las realizan: }\end{array}$ & Cantidad & Porcentaje & Cantidad & Porcentaje & Cantidad & Porcentaje \\
\hline $\begin{array}{l}\text { Hombres y mujeres de } \\
\text { manera igualitaria }\end{array}$ & 30 & $35.7 \%$ & 9 & $18 \%$ & 73 & $46.7 \%$ \\
\hline $\begin{array}{l}\text { Hombres y mujeres } \\
\text { pero más las mujeres }\end{array}$ & 26 & $30.9 \%$ & 14 & $28 \%$ & 35 & $22.4 \%$ \\
\hline $\begin{array}{l}\text { Hombres y mujeres } \\
\text { pero más los hombres }\end{array}$ & - & $0 \%$ & - & $0 \%$ & - & $0 \%$ \\
\hline $\begin{array}{l}\text { Solo los mujeres de la } \\
\text { casa }\end{array}$ & 11 & $13 \%$ & 18 & $36 \%$ & 34 & $21.7 \%$ \\
\hline $\begin{array}{l}\text { Solo los hombres de la } \\
\text { casa }\end{array}$ & - & $0 \%$ & - & $0 \%$ & - & $0 \%$ \\
\hline $\begin{array}{l}\text { No aplica, solo vivimos } \\
\text { mujeres en casa }\end{array}$ & 17 & $20.2 \%$ & 9 & $18 \%$ & 14 & $8.9 \%$ \\
\hline
\end{tabular}

Fuente: Elaboración propia

Para este caso, son las estudiantes de la ENSJ las que señalan que las tareas del hogar se las cargan más a las mujeres y las de la UdeG, hablan de un trato más igualitario. Además, se reitera que los hombres, por sí solos, no se involucran en las tareas hogareñas.

\section{Discusión}

Las chicas que participaron del presente estudio, en su mayoría, son jóvenes de entre 18 y 25 años $(90 \%)$. Quizá sea la razón por la que $93 \%$ de ellas se informó de las manifestaciones por medio de las redes sociales y es que, como plantea Esquivel (2019), la discusión política se facilita por las nuevas formas sociales de relación por medio de Internet y las redes sociodigitales.

En cuanto a la forma de participación de las encuestadas, tenemos que más de $80 \%$ lo hizo de alguna manera. Destaca que $29 \%$ lo hizo por medio de redes sociales. En este sentido, Villacampa y Aran-Ramspott (2020) señalan que las generaciones jóvenes, al integrar las plataformas digitales a sus quehaceres cotidianos, son sensibles a los problemas de violencia de género, su prevención y visibilización. 


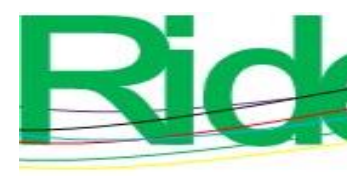

Revista Iberoamericana para la Investigación y el Desarrollo Educativo ISSN 2007 - 7467

En relación con la presencia de la violencia y discriminación hacia las mujeres en las IES, el $64 \%$ manifestaron que si se presenta y las prácticas más reiteradas son la utilización del lenguaje machista, el acoso sexual y la agresión psicológica, tanto de parte de estudiantes varones como de autoridades escolares, aunque se debe resaltar que las menciones bajaron sensiblemente en la situación de las clases en línea con motivo del confinamiento. Al respecto Mingo (2020), señala que la violencia contra las mujeres son prácticas consuetudinarias en las universidades mexicanas no obstante que a nivel discursivo se les reprueba.

En cuanto a la violencia intrafamiliar, al trato que reciben hijas e hijos y a la carga y distribución del trabajo doméstico como resultado del confinamiento, las estudiantes mayoritariamente mencionan que no existe violencia en casa ( $83 \%)$, aunque $12 \%$ señala que es ejercida por el papá o los hermanos varones. Respecto al trato que dan los padres a hombres y mujeres, $70 \%$ señala que es igualitario, pero $27 \%$ reconoce que se da preferencia a hombres. Por último, en lo que concierne a quiénes realizan las tareas domésticas, únicamente $26 \%$ dice que lo hacen hombres y mujeres por igual, $30 \%$ menciona que trabajan más las mujeres y $19 \%$ que exclusivamente lo hacen las mujeres.

Los datos anteriores dan sustento a lo planteado por Bourdieu (2000), quien habla de la existencia de la dominación masculina y que esta se concreta mediante la violencia simbólica, que implica adhesión del dominado, en este caso dominadas, a los requerimientos de quien domina, de tal suerte que existe aceptación tácita de dominación y como corolario sumisión. Solo así se entiende que, por un lado, una gran proporción señale que no existe violencia o que se da un trato igualitario y que, por el otro, una considerable proporción de chicas reconozca que los hombres tienen preferencias o que están exentos de las tareas domésticas en función de su sexo.

Lamas (1994) también está de acuerdo en la vigencia de la dominación masculina y que, en consecuencia, existen tareas que son asignadas de acuerdo con las características atribuidas a los sexos, gracias a lo cual se han construido estereotipos, es decir, ideas socialmente fabricadas. En esto concuerda con lo dicho por Buquet et al. (2013) cuando plantean la necesidad de una transformación cultural a fin de que las tareas de casa sean de corresponsabilidad y no tareas solo de mujeres. 


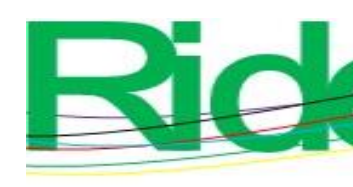

Revista Iberoamericana para la Investigación y el Desarrollo Educativo ISSN 2007 - 7467

\section{Conclusiones}

Respecto a la pregunta de investigación que se planteó, a saber: “¿cómo se posicionan las estudiantes de las instituciones de educación superior de Guadalajara ante las reivindicaciones de las mujeres por sus derechos en el marco de las protestas de marzo del 2020?", encontramos que las chicas se informan, sobre todo, por medio de las redes sociales. Además, casi cuatro de cada cinco participaron de alguna manera en la difusión o de manera directa en las manifestaciones del 8 y 9 de marzo de 2020; sin embargo, existen variables de participación por institución educativa, ya que las estudiantes de la UdeG son las más participativas (90 \%) y las menos, las de UPN (70 \%).

En cuanto a la discriminación y violencia en sus instituciones educativas, se advierte que es alto en condiciones de presencialidad, pues lo refieren dos tercios de las participantes; en las clases en línea bajan, de manera sensible, las agresiones referidas. Pero es de hacer notar que por institución educativa los números cambian, ya que $79 \%$ de las estudiantes de la UdeG se sienten discriminadas o violentadas; en el caso de la ENSJ, el porcentaje baja a $70 \%$, y en el de la UPN a $20 \%$. Para el caso de las clases en línea, los números bajan sensiblemente en las tres instituciones y en la UPN desaparece la discriminación y violencia

Además, la mayoría de estudiantes niega que se presente la violencia intrafamiliar en sus hogares; pero una proporción considerable de chicas considera que se les da mejor trato en el seno familiar a hijos varones sobre las mujeres. Igualmente, la mayoría de estudiantes universitarias y normalistas advierte que las mujeres son quienes se encargan de las tareas domésticas en tiempos de confinamiento.

En cuanto a la hipótesis que se formuló, a partir de que se encontró que más de $90 \%$ dijo haber participado de alguna manera en las jornadas de luchas feministas del 8 y 9 de marzo de 2020, se concluye que sí están informadas sobre sus derechos como mujeres.

De la misma manera, debido a que el número de menciones que se presenta sobre la utilización de lenguaje machista, acoso sexual, agresión psicológica e incluso física es alto (solo baja en condiciones de clases virtuales), se puede decir que la mayoría está en contra de estas prácticas y, por tanto, reivindican su derecho a ser consideradas como iguales respecto a los estudiantes varones. En cuanto al trato que reciben en casa, nos encontramos con cierto nivel de contradicción, ya que un alto porcentaje dice que recibe un trato igualitario con respecto a los varones, pero las menciones sobre quién es mejor tratado y 

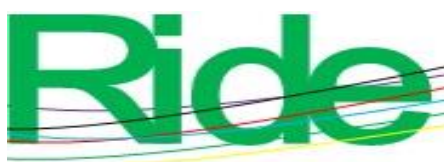

Revista Iberoamericana para la Investigación y el Desarrollo Educativo

ISSN $2007-7467$

quiénes hacen el quehacer en casa ponen en desventaja a las mujeres, por lo que se puede aseverar que no identifican con claridad cómo debe darse el trato de iguales.

\section{Futuras líneas de investigación}

La investigación realizada permitió identificar algunos aspectos a desarrollar para complementar el trabajo presentado, que no se abordaron en este espacio por no estar previamente contemplados: $a$ ) indagar respecto a la posible existencia de colectivas de chicas estudiantes de las instituciones de educación superior en la promoción y defensa de sus derechos y reivindicaciones, $b$ ) conocer la opinión de los estudiantes varones de las instituciones de educación superior con respecto a las demandas feministas y sobre el concepto de dominación masculina y c) conocer la posición y las acciones de las autoridades escolares (de las universidades y escuelas normales) sobre las violencias a las que están sujetas las estudiantes en las instituciones escolares y si existen acciones institucionales para la promoción de la perspectiva de género.

\section{Referencias}

Bourdieu, P. (2000). La dominación masculina. Barcelona, España: Anagrama.

Buquet, A., Cooper, J., Mingo, A. y Moreno, H. (2013). Intrusas en la universidad. México: Universidad Nacional Autónoma de México.

Castro, D. (2020). Jóvenes universitarios y violencia sexual: las tramas del discurso sexista. (Tesis de maestría). Universidad de Chile. Recuperado de http://repositorio.anid.cl/bitstream/handle/10533/241967/Castro\%2C\%20Diego\%20

\%20Sexismo\%20y\%20violencia\%20sexual\%20\%28Tesis\%20Mag\%C3\%ADster\% 29.pdf? sequen.

De Gouges, O. (1791). Declaración de los derechos de la mujer y de la ciudadana. http://clio.rediris.es/n31/derechosmujer.pdf

Esquivel, D. (2019). Construcción de la protesta feminista en hashtags: aproximaciones desde el análisis de redes sociales. Comunicación y Medios $N^{\circ} 40$, pp. 184-198. DOI $10.5354 / 0719-1529.2019 .53836$ 

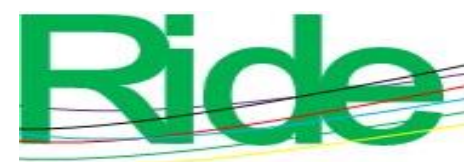

Revista Iberoamericana para la Investigación y el Desarrollo Educativo

ISSN $2007-7467$

Lamas, M. (1994). Cuerpo: diferencia sexual y género. Debate Feminista, (10). Recuperado de https://www.icmujeres.gob.mx/wp-content/uploads/2020/05/2.-CuerpoDiferencia-sexual-y-genero.pdf.

Mingo, A. (2020). "Juntas nos quitamos el miedo". Estudiantes feministas contra la violencia sexista. Revista Interamericana de Educación Superior, 11(31), 3-23. Recuperado de https://doi.org/10.22201/iisue.20072872e.2020.31.703.

Organización de las Naciones Unidas [ONU]. (1976). Informe de la Conferencia Mundial del Año Internacional de la Mujer. Nueva York, Estados Unidos: Organización de las Naciones Unidas. Recuperado de http://ungoals.org/documents/1975\%20world\%20conference\%20on\%20internationa 1\%20womens\%20year\%20Mexico\%20conference\%20report\%20optimized.pdf.

Topete, C. (20 de febrero de 2020). ¿De dónde surge el movimiento \#UnDíaSinNosotras? El Informador. Recuperado de https://www.informador.mx/jalisco/De-donde-surgeel-movimiento-UnDiaSinNosotras-20200220-0108.html.

Torrado, M. (2009). Estudios de caso. En Bisquerra, R. (coord.), Metodología de la investigación educativa (pp. 231-257). España: Editorial La Muralla.

Villacampa, E. y Aran-Ramspott, M. (2020). Jugando a ser youtubers: prácticas digitales para la prevención de la violencia de género. Zer, 25(48), 287-308. Recuperado de https://doi.org/10.1387/zer.21570. 